\title{
Artelogie
}

Recherche sur les arts, le patrimoine et la littérature de l'Amérique latine

$10 \mid 2017$

Après le paysage : l'art, l'inscription et la représentation de la nature en Amérique latine aujourd'hui

\section{Paysage dans les Amériques. Peintures de la Terre de feu à l'Arctique de Peter John Brownlee, Valérica Piccoli, Georgiana Uhlyarik}

\section{Catalina Valdés}

URL : http://journals.openedition.org/artelogie/935

DOI : $10.4000 /$ artelogie.935

ISSN : 2115-6395

Éditeur

Association ESCAL

Référence électronique

Catalina Valdés, « Paysage dans les Amériques. Peintures de la Terre de feu à l'Arctique de Peter John Brownlee, Valérica Piccoli, Georgiana Uhlyarik», Artelogie [En ligne], 10 | 2017, mis en ligne le 05 avril 2017, consulté le 23 septembre 2020. URL : http://journals.openedition.org/artelogie/935 ; DOI : https://doi.org/10.4000/artelogie.935

Ce document a été généré automatiquement le 23 septembre 2020. 


\title{
Paysage dans les Amériques. Peintures de la Terre de feu à l'Arctique de Peter John Brownlee, Valérica Piccoli, Georgiana Uhlyarik
}

\author{
Catalina Valdés
}

\section{RÉFÉRENCE}

Peter John Brownlee, Valérica Piccoli, Georgiana Uhlyarik, Paisaje en las Américas.

Pinturas desde la Tierra del Fuego al Ártico, Toronto, Art Gallery of Ontario, Pinacoteca do Estado de São Paulo, Terra Foundation \& Yale University Press, 2015, 280p.

1 L'idée d'une Amérique continentale semble diffuse lorsque nous pensons en termes géopolitiques et nationaux. Cet ouvrage n'a pas pour but de résoudre les ambiguïtés culturelles ou clore les conflits historiques, pas davantage d'exagérer les fraternités ni dénoncer les hégémonies. Il offre en revanche une vision panoramique construite à partir d'une idée forte : la représentation culturelle de la nature - la construction de paysages - est une geste commune dont l'impact est profond dans l'histoire de la visualisation moderne d'un continent qui, selon les auteurs, s'appréhende mieux de façon plurielle. Il propose aussi un exercice autoral collectif comme une façon de préserver la multiplicité de langues et de perspectives, en esquissant concrètement une histoire transnationale qui ne débouche pas forcément sur une histoire commune, dans la mesure où elle s'appuie sur des études de cas. Plutôt qu'un récit unique, l'ouvrage propose six axes, ou constellations autour desquels prennent place les nombreux travaux et multiples visions des chercheurs conviés. Ces axes rendent compte d'une conception complexe du paysage qui dépasse largement les idées esthétiques du pittoresque et du sublime, parce que la dimension scientifique et économique est présente dans ses multiples représentations, s'agissant d'un contexte marqué par les 
processus de découverte et d'exploitation du territoire, en soulignant les avancées politiques et sociales du paysage en tant que genre.

2 Cette publication est la somme d'un projet de recherche de grande ampleur sur la présence de la nature dans la culture visuelle des Amériques, particulièrement sur le genre pictural du paysage, du début du XIXe siècle jusqu'au début du XXe siècle. Un projet de cette envergure n'est compatible qu'à partir d'un travail de collaboration et tel a été le cas des auteurs et commissaires d'exposition de trois centres d'art prestigieux au sein du continent, John Peter Brownlee pour la Fondation Terra de Chicago, Valeria Piccoli pour la pinacothèque de l'État de São Paulo et Georgiana Uhlyarik, pour la Galerie d'art de l'Ontario. Ces travaux sont redevables envers d'innombrables collaborations de spécialistes de l'histoire de l'art dans plusieurs pays d'Amérique, ont donné lieu à une série de rencontres académiques sur/autour du thème et auront culminé avec l'exposition itinérante homonyme, inaugurée en la Galerie d'Art de l'Ontario en juin 2015, en coïncidence avec les Jeux Panaméricains, présentée ensuite au Crystal Bridges Museum of American Art (Arkansas) la même année et enfin, à la Pinacothèque de São Paulo début 2016. L'ouvrage a été édité en anglais, en espagnol et en portugais. Il est, en outre, possible d'avoir accès à l'exposition virtuelle sur le site : http://picturingtheamericas.org/themes/?lang=fr

\section{AUTEURS}

\section{CATALINA VALDÉS}

docteur en Histoire de l'art, CRAL- EHESS/ IDAES-UNSAM

cvaldese[at]gmail.com 